\title{
A Fatal Case of Melioidosis in a Patient with Chronic Pancreatitis
}

\author{
Shivangi Mishra*, Padmakumar V Arayamparambil, Garud Suresh Chandan, Pooja Prathapan Sarada
}

Fortis Hospital Bannerghatta Road, 154, 9, Bannerghatta Main Rd, Opposite IIM, Sahyadri Layout, Panduranga Nagar, Bengaluru, Karnataka 560076, India

DOI: $10.36347 /$ sjmcr.2020.v08i07.008

| Received: 08.07.2020 | Accepted: 15.07.2020 | Published: 18.07.2020

*Corresponding author: Dr. Shivangi Mishra

Abstract

Case Report

Melioidosis is a systemic infection caused by gram negative bacteria Burkholderia pseudomallei which spreads through contact with soil and water. High endemicity of Melioidosis is seen in tropical countries in south east Asia specially Thailand, Malaysia, Singapore and Australia. Melioidosis is relatively rare in India and usually presents with systemic symptoms, multiple abscess, consolidation and septic shock. We report a case of meliodosis in a metropolitan city in South India presenting initially with chronic pancreatitis. Difficulty in identification and a low index of suspicion of Burkholderia pseudomallei caused delayed reporting of the species. Proven cases of Meliodosis in India could just be the tip of the iceberg. Varied presentation, its misdiagnosis as other species and the high intrinsic resistance has attributed to its high mortality. With sporadic cases being reported from non endemic areas, a high index of suspicion and early initiation of treatment may save lives.

Keywords: Melioidosis, burkholderia, pancreatitis.

Copyright @ 2020: This is an open-access article distributed under the terms of the Creative Commons Attribution license which permits unrestricted use, distribution, and reproduction in any medium for non-commercial use (NonCommercial, or CC-BY-NC) provided the original author and source are credited.

\section{INTRODUCTION}

Melioidosis or Whitmores disease is a chronic systemic infection caused by gram negative saprophytic bacteria Burkholderia pseudomallei. This bacterium usually spreads through contact with soil and water and is highly endemic in tropical countries specially Thailand, Malaysia, Singapore and Australia [1]. Though relatively rarely reported in India, recently certain parts of India like Tamil Nadu and South western costal regions have been declared endemic. Some sporadic cases have been reported from Kerala, Maharashtra, Andhra Pradesh and Tripura [1]. Melioidosis presents with a wide spectrum of systemic symptoms. It can range from multiple cutaneous and visceral abscess, consolidation, encephalitis, septic arthritis to overt sepsis and septic shock. The mortality in patients with Meliodosis is more than $40 \%$. Mortality in patients with Melioidosis and septic shock is upto 80$100 \%$ [2]. We report a case of Melioidosis in a metropolitan city in South India in a patient with chronic pancreatitis and diabetes.

\section{Case Report}

A 28 year old male presented with a five days history of fever. He was initially evaluated at a local centre, where he was found to be thrombocytopenic. He tested negative for Dengue and malaria. In view of persistent fever, he was referred to our setup for further evaluation. He was a known case of chronic pancreatitis diagnosed seven years back and had necrosectomy and cystogastrostomy for pancreatic necrosis and pseudocyst. He was recently diagnosed with diabetes mellitus and hypertension and was on oral hypoglycaemic and anti hypertensive agents. He had recent history of travel to Tirupati, which is non endemic for Melioidosis. On arrival to our hospital, cultures and throat swab were sent and he was started on Piperacillin Tazobactum and Azithromycin as per our hospital protocol. In view of type 1 respiratory failure after initial trial of non invasive ventilation, he was intubated and mechanically ventilated. Chest $\mathrm{X}$ ray showed bilateral minimal infiltrates and lung ultrasound showed a shred sign. His blood culture was reported initially as gram negative bacilli. His throat swab tested negative for viral infection. He developed multiorgan failure requiring multiple vasopressor supports, renal replacement therapy and escalation of antibiotic to Carbapenem. By day five he was requiring triple vasopressor support and was in severe ARDS. Blood culture was reported on day five as Burkholderia pseudomallei, sensitive to Cotrimoxazole only. He was started on Cotrimoxazole. Unfortunately, he succumbed to death due to multi organ dysfunction and septic shock. 


\section{DISCUSSION}

The first case of Melioidosis was reported in India in 1953 [3]. Since then multiple cases of Melioidosis have been reported mostly from south western costal belts and Tamil Nadu. Few cases have been reported from Kerala, Maharashtra, central India, West Bengal and Tripura.[1] The reported cases were in patients with contact to environment including soil, polluted rain water, rice fields, trauma and abrasions and agricultural workers. Incidence of Melioidosis is higher in men, probably because of increased occupational exposure [2-5]. Other risk factors include diabetes mellitus, chronic kidney disease and immunocompromised states [2]. Melioidosis has a wide range of presentation and clinical manifestation making the diagnosis difficult. Most commonly reported symptoms are septic arthritis, pulmonary consolidation, cutaneous and visceral abscesses, hepatosplenomegaly $[1-3,5,6]$. There are reports of presentation just with acute febrile illness as in our patient [2]. There have been reports of delayed presentation long time after exposure [3,4]. The longest latent period documented is 62 years. A study done in endemic population in India shows a $29 \%$ seropositivity [7].

Burkholderia pseudomallei is commonly reported as Pseudomonas or non fermenting gram negative bacteria in resource limited setting [2, 8]. Species level identification of Burkholderia is imperative as it has significant impact on treatment. Burkholderia pseudomallei is commonly responsive to Cotrimoxazole and Doxycycline. It is intrinsically resistant to Polymyxins, while Pseudomonas is intrinsically resistant to cotrimoxazole and responds to Polymixins [2, 8]. Isolation in blood culture is the gold standard for diagnosis [8]. In our setup, Burkholderia was isolated on blood agar. Species identification of Burkholderia pseudomallei was done using VITEK 2. The usual recommended therapy for meliodosis consists of an acute intensive phase and an eradication phase. Acute intensive phase is of two weeks of intravenous Ceftazidime or Carbapenem, with Meropenem having lower MIC than Imipenem. Oral cotrimoxazole should be continued for around 20 weeks as eradication phase $[2,8,9]$. Our patient was already on Meropenem, hence Cotrimoxazole was added.

\section{CONCLUSION}

The wide range of symptoms, difficulty or misdiagnosis during culture, delayed reporting of culture results and high levels of intrinsic resistance lead to high morbidity and mortality in Melioidosis. A high index of suspicion helps in saving patients life. Difficulty in diagnosis, misdiagnosis as Pseudomonas and inadequate experience of laboratories with the organism may lead to under-reporting of the cases of Melioidosis and hence leads to underestimation of the global burden of the disease.

This article highlights the possible under reporting of cases of Melioidosis and the high probability of other geographical areas affected by this infection which may also be endemic. The number of cases of Melioidosis identified and reported are probably just the tip of an iceberg. Melioidosis could have been misdiagnosed in many cases where lives could have been saved.

\section{REFERENCES}

1. Mukhopadhya A, Balaji V, Jesudason MV, Amte A, Jeyamani R, Kurian G. Isolated liver abscesses in melioidosis. Indian Journal of Medical Microbiology. 2007;25:150.

2. Fatema K, Ahsan AA, Barai L, Saha DK, Afroze SR, Faruq MO, Ahmed F, Saha M. A case of fatal meliodosis presenting as septic arthritis and septicaemia. Bangladesh Critical Care Journal. 2015 Sep 22;3(2):74-6.

3. Ives JCJ, Thomson TJ. Chronic Meliodosis: The First Report of a Case Infected in Central India. Glasgow Med J. 1953; 34:61-7.

4. Deris ZZ, Hasan H, Siti Suraiya MN. Clinical characteristics and outcomes of bacteraemic melioidosis in a teaching hospital in a northeastern state of Malaysia: a five-year review. J Infect Dev Ctries. 2010;4:430-5.

5. Barman P, Kaur R, Kumar K. Clinically lesser known entity in India: A Report of two cases of Melioidosis. Indian J Crit Care Med. 2013;17:468.

6. Jagtap N, Shah H, Kancharla A, Tandan M, Pal P, Lakhtakia S, Ramchandani M, Reddy DN. Gastrointestinal manifestations of melioidosis: A single center experience. Indian Journal of Gastroenterology. 2017 Mar 1;36(2):141-4.

7. Vandana KE, Mukhopadhyay C, Tellapragada C, Kamath A, Tipre M, Bhat V, Sathiakumar N. Seroprevalence of Burkholderia pseudomallei among adults in coastal areas in southwestern India. PLoS neglected tropical diseases. $2016 \mathrm{Apr}$ 14;10(4):e0004610.

8. Princess I, Ebenezer R, Ramakrishnan N, Daniel AK, Nandini S, Thirunarayan MA. Melioidosis: An Emerging Infection with Fatal Outcomes. Indian J Crit Care Med. 2017;21:397-400.

9. Ganesan V, Sundaramoorthy R, Subramanian S. Melioidosis-Series of Seven Cases from Madurai, Tamil Nadu, India. Indian J Crit Care Med. 2019;23:149-51. 